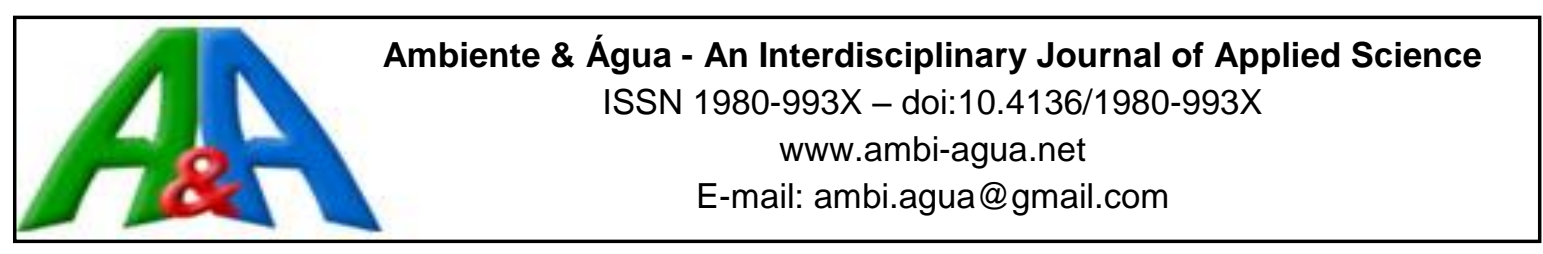

\title{
Análise espacial da ocorrência de leishmaniose visceral no estado do Tocantins, Brasil
}

\author{
doi:10.4136/ambi-agua.1870
}

Received: 2 Oct. 2016; Accepted: 12 Nov. 2016

\author{
Iolanda Graepp Fontoura ${ }^{1,2}$; Volmar Morais Fontoura ${ }^{1,3}$; \\ Luiz Fernando Costa Nascimento ${ }^{1^{*}}$ \\ ${ }^{1}$ Universidade de Taubaté (UNITAU), Taubaté, SP, Brasil \\ Programa de Pós-Graduação em Ciências Ambientais \\ ${ }^{2}$ Universidade Federal do Maranhão \\ ${ }^{3}$ Universidade Estadual do Tocantins \\ *Autor correspondente: e-mail: luiz.nascimento@unitau.com.br, \\ iolandagraepp@hotmail.com, volmar_morais@hotmail.com
}

\section{RESUMO}

O Brasil e outros quatro países detêm $90 \%$ dos casos de leishmaniose visceral, que é uma doença grave que acarreta óbito, se não tratada. Este estudo teve por objetivo identificar padrões espaciais de distribuição da leishmaniose visceral no estado do Tocantins, Brasil, de 2008 a 2011. Trata-se de estudo ecológico e exploratório com dados obtidos do Datasus e realizada análise por município. Foram estimados os índices de Moran global e construídos mapas temáticos das taxas por 100 mil habitantes, mapa de Moran e de Kernel. Foram georreferenciados 1778 casos de leishmaniose visceral representando uma taxa de 31,75 casos/100 mil habitantes sendo a taxa variou entre 0,00 e 343,16 por 100.000 habitantes com a microrregião de Araguaína, no norte do estado, a mais atingida. O índice de Moran foi $\mathrm{Im}=$ 0,20 ( $\mathrm{p}$-valor < 0,01). Constatou-se que as microrregiões com maior necessidade de intervenção são as de Araguaína e do Bico do Papagaio, onde foi identificada maior densidade de casos notificados por local de residência, assim como os municípios vizinhos a Juarina, pertencentes à microrregião de Miracema do Tocantins.

Palavras-chave: análise espacial, leishmaniose visceral, sistemas de informação geográfica.

\section{Spatial analysis of the occurrence of visceral leishmaniosis in the state of Tocantins, Brazil}

\section{ABSTRACT}

Brazil and other four countries account for $90 \%$ of the cases of visceral leishmaniosis, which is a serious disease that causes death if untreated This study aimed to identify spatial patterns of visceral leishmaniosis distribution in the state of Tocantins, Brazil, from 2008 to 2011. It is an ecological and exploratory study that performed analysis by municipality using data obtained from Datasus. The global Moran indices were estimated and thematic maps of the rates per 100000 inhabitants were constructed, Moran and Kernel map. There were 1778 georeferenced cases of visceral leishmaniosis representing a rate of 31.75 cases / 100000 inhabitants and the rate ranged between 0.00 and 343.16 per 100,000 population with the microAraguaína, in the northern state, the most affected. Moran's index was $\operatorname{Im}=0.20(\mathrm{p}<0.01)$. It 
was found that the micro-regions most in need of intervention are the Araguaína and Parrot's Beak, which showed an increased density of cases reported by place of residence, as well as neighboring municipalities of Juarina in the micro-region of Miracema do Tocantins.

Keywords: geographic information system, spatial analysis, visceral leishmaniosis.

\section{INTRODUÇÃO}

A leishmaniose visceral (LV) é uma doença de grande significância epidemiológica por envolver espécies diferentes de parasitas Leishmania, comportamentos de vetores flebotomíneos, como o Lutzomyia longipalpis, hospedeiros e reservatórios em ambos os ciclos de transmissão (Dantas-Torres et al., 2006; Almeida et al., 2010). O cão doméstico é um importante reservatório e a forma de transmissão se faz pela picada do vetor Lutzomyia longipalpis infectado ou por via transfusional. É possível observar um crescente aumento de casos de leishmaniose visceral. Esta pode ser classificada de moderada a grave, geralmente caracterizada por febre, palidez cutânea e hepatoesplenomegalia, podendo vir acompanhada de diarreia e tosse. Os indivíduos mais acometidos são as crianças, principalmente as menores de 1 ano, e a doença pode estar associada a outros processos infecciosos, inclusive o vírus da imunodeficiência adquirida humana (HIV). Geralmente, a leishmaniose visceral é fatal se não tratada. Estima-se que haja 350 milhões de pessoas no mundo em risco de contrair leishmaniose, em suas diferentes formas clínicas, e que ocorram dois milhões de casos anualmente sendo que, entre 500.000 casos da doença, cerca de 59.000 pessoas morrem. Trata-se de uma doença endêmica em muitos países, como a Índia, o Nepal, Bangladesh, o Sudão e o Brasil, sendo este último o responsável pela maioria dos casos na América do Sul (WHO, 2010).

Neste continente, situações epidemiológicas, socioeconômicas e processos ecológicos podem reduzir o impacto dos programas de controle. No Brasil, são notificados a cada ano cerca de 3.500 casos de leishmaniose visceral (Dantas-Torres et al., 2006). Nos últimos anos foram notificados cerca de 40.500 casos e esse número tem aumentado ao longo dos anos (Santos et al., 2003). Entre os estados mais acometidos encontram-se Maranhão, Minas Gerais, Ceará, Tocantins, Bahia, Piauí, Pará, Mato Grosso do Sul e São Paulo (Brasil, 2016a). A expansão desta doença vem ocorrendo no espaço e no tempo, com aumento do número de municípios afetados e do número de casos e óbitos (Cardim et al., 2013). O uso de Sistemas de Informação Geográfica (SIG) e ferramentas de análise espacial permite analisar esse processo, possibilitando compreender os padrões espaciais da distribuição de um desfecho, bem como identificar áreas de risco e possíveis fatores associados e indicar áreas prioritárias para desenvolvimento de ações de vigilância e controle. (Nascimento et al., 2007; Carvalho e Nascimento, 2012; Venâncio et al., 2015).

Esta pesquisa teve por objetivo identificar padrões espaciais da distribuição da leishmaniose visceral no estado do Tocantins, de 2008 a 2011, bem como identificar áreas com altas densidades de taxas de leishmaniose visceral e observar a existência de autocorrelação global e local da ocorrência da leishmaniose visceral com possíveis fatores associados como focos de queimadas, dados de coleta de lixo e saneamento básico.

\section{MATERIAL E MÉTODOS}

Realizou-se um estudo ecológico e exploratório, com dados do Departamento de Informática do SUS - DATASUS, disponibilizados pelo SINANET (Brasil, 2016b) utilizando as ferramentas de geoprocessamento para identificar a distribuição espacial dos casos de leishmaniose visceral, analisados sob a forma de taxas, no estado de Tocantins, em seus 139 municípios, de 2008 a 2011. Tocantins está dividido em 8 microrregiões, Bico do Papagaio, Araguaína, Miracema do Tocantins, Jalapão, Porto Nacional, Rio Formoso, Gurupi, Dianópolis, 
ocupa uma área de $277.620 \mathrm{~km}^{2}$ e sua população é de cerca de 1,400 milhão (Figura 1) (IBGE, 2016).

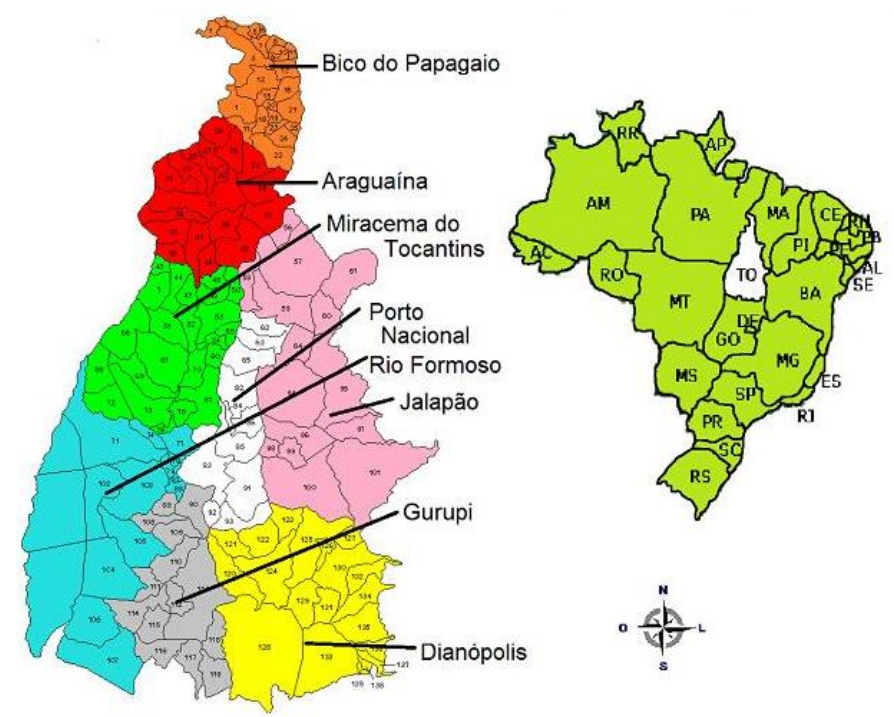

Figura 1. Estado do Tocantins. Sua localização no Brasil e suas microrregiões.

Para a análise estatística espacial foi utilizado o programa computacional TerraView, versão 4.0.0 e estimados os índices de Moran global; este índice é definido no intervalo de -1 a 1. Quanto mais próximo de 1 representa dependência espacial positiva, quanto mais próximo de zero significa ausência de dependência espacial e mais próximo a -1 é dependência espacial negativa. Foi utilizado o índice de Moran local que permite identificar aglomerados e também municípios que necessitam de uma intervenção. Foram estudados todos os casos de leishmaniose visceral, notificados nos 139 municípios do estado, por local de residência. Os dados de cada ano foram comparados, bem como as variáveis e possíveis interações com o meio ambiente de cada município, como focos de queimadas obtidos do portal do CPTEC-INPE (2016), dados de coleta de lixo e saneamento básico (Brasil, 2106c). Foram construídos mapas temáticos com as taxas por 100 mil habitantes, o mapa de Moran que identifica municípios com alta prioridade de intervenção e mapa de Kernel que é um método estatístico de estimação de curvas de densidades sendo que cada uma das observações é ponderada pela distância em relação a um valor central, o núcleo.

Como foram analisados quatro anos consecutivos, é possível que isso tenha minimizado oscilações aleatórias.

\section{RESULTADOS E DISCUSSÃO}

Foram registrados 1.778 casos de leishmaniose visceral no período de estudo, entre 2008 e 2011, representando uma taxa de 33,42 casos por 100.000 habitantes. O índice de Moran global detectou grau de dependência espacial no coeficiente de autocorrelação no conjunto de dados. $O$ valor do índice foi de $\mathrm{I}_{\mathrm{m}}=0,20$ e p-valor $<0,01$.

Ao longo dos quatro anos estudados, foi possível observar que houve uma diminuição do número de óbitos, porém, houve um aumento do número de casos, bem como a expansão da doença, pois em 2008 a densidade de casos era maior na microrregião do Bico do Papagaio e em 2011 isso ocorreu na microrregião de Araguaína. Tal fato se deve ao possível fluxo migratório intermunicipal, colaborando com a disseminação do agente gerador da doença para municípios vizinhos (Dantas-Torres e Brandão-Filho, 2006). 
Em relação ao gênero, 878 indivíduos (57\%) eram do sexo masculino e 655 (43\%) eram do sexo feminino. Esse fato, provavelmente, decorre do tipo de trabalho que desenvolvem, em locais como currais e terrenos baldios, atuando como vaqueiros ou lixeiros, entre outros; os homens costumam expor mais seu corpo.

Em relação à faixa etária, observou-se que 230 (13\%) eram crianças menores de 1 ano e $626(35 \%)$ tinham de 1 a 4 anos. Provavelmente, essa ocorrência deve-se à baixa imunidade e com essa idade as crianças gostam de brincar no entorno do domicílio, onde, geralmente, podem ser encontrados os criadouros do mosquito palha (Mestre e Fontes, 2007).

Em relação ao nível de escolaridade, o maior número foi de $362(21 \%)$, equivalente às pessoas com o nível de escolaridade até o $4^{\circ}$ ano do Ensino Fundamental; possivelmente, isso se relaciona ao fato de que, devido à sua baixa escolaridade, esses indivíduos se dispõem a atuar em funções para as quais não se exige formação profissional, como vaqueiros, lixeiros, catadores de lixo, garis etc., e em locais propícios para criadouros do mosquito palha.

A leishmaniose visceral está distribuída por todo o estado do Tocantins, porém, os maiores coeficientes encontram-se nas microrregiões de Araguaína e Bico do Papagaio, que se localizam no norte do estado. Dos 139 municípios, 97 (69,78\%) notificaram casos de leishmaniose visceral. Em referência ao coeficiente de detecção, a amplitude variou entre 0 e 343,16 por 100.000 habitantes, como mostra a Figura 2, sendo que, destes, 46 municípios $(47,42 \%)$ apresentaram nível elevado de detecção, superior ao valor encontrado em todo estado do Tocantins. Destacam-se três municípios com taxas elevadas, a saber: Araguaína (343,16/100 mil hab; Araguatins (152,71/100 mil hab e Ananás (123,69/100 mil hab).

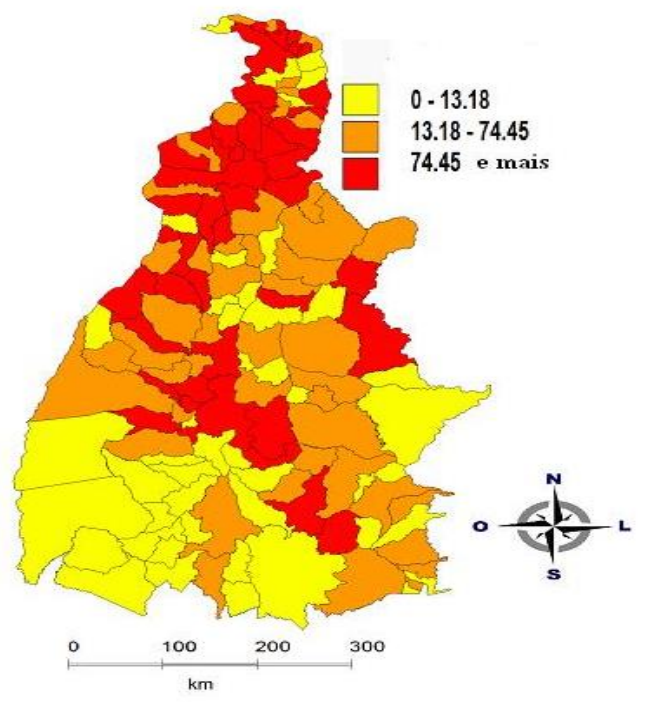

Figura 2. Taxas de leishmainose visceral, por 100 mil habitantes, nos municípios de Tocantins, BR, 20082011.

Por meio do Moran Map, exposto na Figura 3, foi possível identificar 13 municípios com maior necessidade de intervenção, referentes à distribuição da leishmaniose visceral no Tocantins entre os anos de 2008 e 2011. Mostra-se possível controlar a leishmaniose visceral em campos endêmicos, para precaver a ativação eu reativação de focos.

Por meio do estimador de densidade de Kernel, foi possível analisar a densidade espacial exploratória do comportamento da classificação espacial de leishmaniose visceral no Tocantins, onde se estimou a magnitude de acontecimento do processo exato em toda a extensão de estudo. Dessa forma, na Figura 4, pôde-se obter uma visão geral da distribuição espacial da ocorrência 
da leishmaniose em toda a área estudada, provendo uma primitiva aproximação da amostra espacial exposta. Determinou-se o nível de risco da doença na área estudada, onde as áreas com maior atividade de casos são realçadas em vermelho, com a "hot spot".

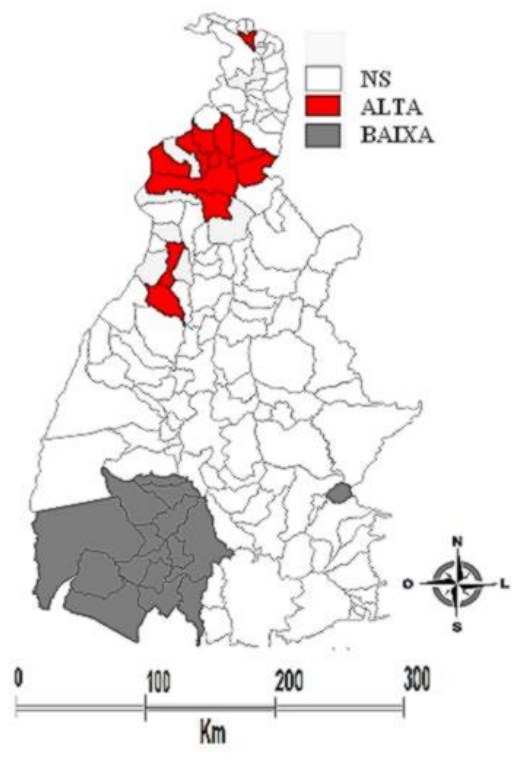

Figura 3. Mapa de Moran identificando municípios com alta e baixa prioridade de intervenção, Tocantins, BR, 2008-2011.

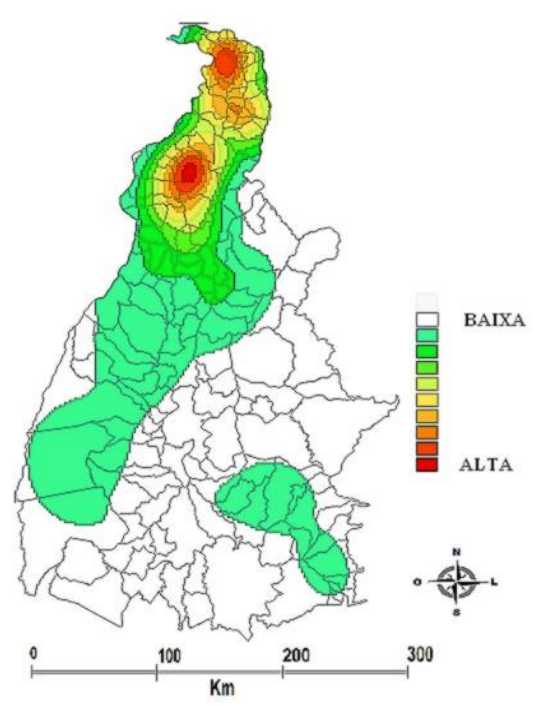

Figura 4. Mapa de Kernel, identificando regiões com densidades, baixa até alta, de casos de leishmaniose visceral, Tocantins, BR, 2008-2011.

Não houve correlação entre as taxas de leishmaniose visceral nos municípios e saneamento básico, focos de queimadas e dados de coleta de lixo. A taxa média encontrada de 33,42/ 100 mil habitantes foi a maior encontrada neste período para os estados brasileiros; o segundo estado 
com maior taxa foi Mato Grosso do Sul com 11,55/ 100 mil habitantes, sendo que nos demais estados as taxas variaram entre 0,02 e 8,29/ 100 mil habitantes (Brasil, 2016b)

As maiores incidências verificadas no sexo masculino e nas crianças entre zero e quatro anos estão de acordo com outros estudos, nos quais a maior incidência nos homens tem sido atribuída a questões ocupacionais e nas crianças, ao desenvolvimento incompleto do sistema imunológico (Cardim et al., 2013; Barata et al., 2013; Botelho et al., 2009; Monteiro et al., 2005; Mestre e Fontes, 2007; Souza et al., 2008).

As maiores taxas encontradas se encontram na região norte e noroeste do estado, na divisa com os estados de Pará e Maranhão, mas há municípios com altas taxas na região central. $\mathrm{O}$ mapa de Kernel confirma as altas taxas por área, nestas regiões.

As menores taxas se encontram na região sudoeste do estado em direção ao estado do Mato Grosso. O mapa de Lisa mostra aglomerado significativo nesta região e o mapa de Moran confirma que é uma região com baixa prioridade para intervenção.

Ao longo dos quatro anos estudados, foi possível identificar um aumento do número de casos notificados. Por meio do índice de Moran global, identificou-se que existe autocorrelação espacial no grau de dependência de 0,20 , bem como se mostrou nível de significância do valor $p$, que foi de 0,01 , indicando similaridade entre municípios vizinhos.

No mapa Moran local, LISA, identificou-se a microrregião de Araguaína, no norte do estado, com maiores taxas e inversamente proporcional à microrregião de Gurupi, no sul do Tocantins, com menores taxas de leishmaniose visceral.

O Moran Map identificou 13 municípios com alta prioridade de medidas de intervenção localizados na região do Bico do Papagaio e de Araguaína onde as taxas atingiram valores da ordem de 300 casos por 100mil habitantes; o mapa de Kernel também aponta esta região com altas densidades.

E no mapa de Kernel se obteve densidades maiores das taxas de leishmaniose visceral na região norte do estado, estimando, assim, a magnitude da ocorrência de casos notificados por local de residência e determinando o nível de risco da doença sobre a área estudada.

Dentre os motivos para estas taxas elevadas podem residir no fluxo de pessoas e mercadorias pelas rodovias e ferrovias que interligam as regiões brasileiras e seus centros urbanos, os processos migratórios, a urbanização e as condições precárias de saneamento, o deslocamento de animais infectados, adaptação do vetor, entre outras, além da necessidade de estruturação das equipes de saúde. (Antonialli et al., 2007, Mestre e Fontes, 2007; Scandar et al., 2011). Outro motivo que poderia explicar estas taxas elevadas seria uma melhor identificação dos casos, bem como uma busca ativa mais eficiente por parte da vigilância epidemiológica do estado.

O transporte de cães infectados e/ou doentes, provenientes das várias regiões brasileiras com transmissão de LV, pode ter contribuído para a introdução e expansão da doença para outras áreas, bem como a circulação de pessoas, animais e produtos entre municípios de uma mesma área endêmica pode ter influenciado a expansão da doença (Mestre e Fontes, 2007). A introdução do parasito em regiões sem transmissão poderia ocorrer pelo envio de cães assintomáticos de outras áreas, a fim de que não fossem eutanasiados (Scandar et al., 2011).

Este estudo pode ter limitações e, dentre elas, o fato de se utilizar dados secundários, apesar de serem obtidos de fontes oficiais; erros no diagnóstico e subnotificação de casos poderiam comprometer as taxas.

Apesar destas possíveis limitações foi possível identificar municípios com taxas elevadas e quais devem ser alvo de intervenção dos gestores municipais.

Assim, mostra-se possível propor medidas de intervenção em determinadas microrregiões para diminuir as taxas de leishmaniose visceral e promover ações de educação ambiental combinadas à educação em saúde. 


\section{CONCLUSÃO}

Este estudo possibilitou a identificação de padrões espaciais da distribuição da leishmaniose visceral no estado do Tocantins. Por meio de mapas temáticos foram identificadas áreas de alta prioridade de intervenção e a densidade de taxas segundo os municípios.

\section{REFERENCIAS}

ALMEIDA, P. S.; NASCIMENTO, J. C.; FERREIRA, A. D.; MINZÃO, L. D.; PORTES, F.; MIRANDA, A. M. et al. Espécies de flebotomíneos (Diptera, Psychodidae) coletadas em ambiente urbano em municípios com transmissão de leishmaniose visceral do estado de Mato Grosso do Sul, Brasil. Revista Brasileira de Entomologia, v. 54, n. 2, p. 304-310, 2010. http://dx.doi.org/10.1590/S0085-56262010000200014

ANTONIAlli, S. A. C.; TORRES, T. G.; PARANHOS Fo, A. C.; TOLEZANO, J. E. Spatial analysis of American Visceral Leishmaniasis in Mato Grosso do Sul State, Central Brazil. Journal of Infectiology, v. 54, n. 5, p. 509-514, 2007. http://dx.doi.org/10.1016/j.jinf.2006.08.004

BARATA, R. A.; PEIXOTO, J. C.; TANURE, A.; GOMES, M. E.; APOLINÁRIO, E. C.; BODEVAN, E. C. et al. Epidemiology of visceral leishmaniasis in a reemerging focus of intense transmission in Minas Gerais State, Brazil. BioMed Research Internationa, v. 2013, p. 6, 2013. http://dx.doi.org/10.1155/2013/405083

BOTELHO, A. C. A.; NATAL, D. Primeira descrição epidemiológica da leishmaniose visceral em Campo Grande, Estado de Mato Grosso do Sul. Revista da Sociedade Brasileira de $\begin{array}{lllllll}\text { Medicina Tropical, } & \text { v. 42, n. } & \text { n, } & \text { p. } & \text { 503-508, }\end{array}$ http://producao.usp.br/handle/BDPI/12682

BRASIL. Ministério da Saúde. Portal da Saúde. DATASUS. Informações de Saúde (TABNET): epidemiológicas e morbidade. Disponível em: https://goo.gl/2d3oLh Acesso em: 30 set. $2016 a$.

BRASIL. Ministério da Saúde. Portal da Saúde. DATASUS. Informações de Saúde (TABNET): indicadores de morbidade. Disponível em: http://tabnet.datasus.gov.br/ cgi/tabcgi.exe?idb2012/d0205. Acesso em: 30 set. 2016.

BRASIL. Ministério da Saúde. Portal da Saúde. DATASUS. Informações de Saúde (TABNET): coleta de lixo. Disponível em: http://tabnet.datasus.gov.br/cgi/ibge/ lixdescr.htm. Acesso em: 29 set. $2016 \mathrm{c}$.

CARDIM, M. F. M.; RODAS, L. A. C. A.; DIBO, M. R.; GUIRADO, M. M.; OLIVEIRA, A. M.; CHIARAVALLOTI NETO, F. Introdução e expansão da leishmaniose visceral americana em humanos no Estado de São Paulo, 1999-2011. Revista de Saúde Pública, v. 47, n. 4, p. 691-700, 2013.

http://dx.doi.org/10.1590/S0034-8910.2013047004454

CARVALHO, R. M.; NASCIMENTO, L. F. C. Spatial distribution of dengue in the city of Cruzeiro, São Paulo state, Brazil: use of geoprocessing tools. Revista do Instituto de Medicina Tropical, v. 54, n. 5, p. 261-264, 2012. http://dx.doi.org/10.1590/S003646652012000500005 
DANTAS-TORRES, F.; BRANDÃO-FILHO, S. P. Expansão geográfica da leishmaniose visceral no estado de Pernambuco. Revista da Sociedade Brasileira de Medicina Tropical, v. 39, n. 4, p. 352-356, 2006. http://dx.doi.org/10.1590/S003786822006000400007

INSTITUTO BRASILEIRO DE GEOGRAFIA E ESTATÍSTICA - IBGE. Censo 2010. Disponível em: http://censo2010.ibge.gov.br/. Acesso em: 13 out. 2016.

INSTITUTO NACIONAL DE PESQUISA ESPACIAL. Centro de Previsão de Tempo e Estudos Climáticos - CPTEC/INPE. Queimadas: monitoramento de focos. Disponível em: http://www.dpi.inpe.br/proarco/bdqueimadas/. Acesso em: 20 set. 2016.

MESTRE, G. L. C.; FONTES, C. J. F. A expansão da epidemia da leishmaniose visceral no estado de Mato Grosso, 1998-2005. Revista da Sociedade Brasileira de Medicina Tropical, v. 40, n. 1, p. 42-48, 2007. http://dx.doi.org/10.1590/S0037-86822007000100008

MONTEIRO, E. M.; SILVA, J. C. F.; COSTA, R. T.; COSTA, D. C.; BARATA, R. A.; PAULA, E. V. et al. Leishmaniose visceral: estudo de flebotomíneos e infecção canina em Montes Claros, Minas Gerais. Revista da Sociedade Brasileira de Medicina Tropical, v. 38, n. 2, p. 147-152, 2005.

http://dx.doi.org/10.1590/S0037-86822005000200004

NASCIMENTO, L. F. C.; BATISTA, G. T.; DIAS, N. W.; CATELANI, C. S.; BECKER, D.; RODRIGUES, L. Análise espacial da mortalidade neonatal no Vale do Paraíba, 1999 a 2001. Revista de Saúde Pública, v. 41, n. 1, p. 94-100, 2007. http://dx.doi.org/10.1590/S0034-89102007000100013

SANTOS, S. O.; ARIAS, J.; HOFFMANN, M. P.; FURLAN, M. B. G.; FERREIRA, W. F.; PEREIRA. C. et al. The presence of Lutzomyia longipalpis in a focus of American visceral leishmaniasis where the only proven vector is Lutzomyia cruzi. Corumbá, Mato Grosso do Sul State. Revista da Sociedade Brasileira de Medicina Tropical, v. 36, n. 5, p. 633-634, 2003. http://dx.doi.org/10.1590/S0037-86822003000500017

SCANDAR, S. A. S.; SILVA, R, A.; CARDOSO Jr., R. P.; OLIVEIRA, F. H. Ocorrência de leishmaniose visceral americana na região de São José do Rio Preto, estado de São Paulo, Brasil. Boletim Epidemiológico Paulista, v. 8, n. 88, p. 13-22, 2011.

SOUZA, C. L. N.; LUZ, Z. P.; RABELLO, A. Análise da informação sobre a leishmaniose visceral disponível em portais brasileiros da rede mundial de computadores: internet. Revista da Sociedade Brasileira de Medicina Tropical, v. 41, n. 4, p. 352-357, 2008. http://dx.doi.org/10.1590/S0037-86822008000400006

VENÂNCIO, T. S.; TUAN, T. S.; NASCIMENTO, L. F. C. Incidência de tuberculose em crianças no estado de São Paulo, Brasil, sob enfoque espacial. Ciência e Saúde Coletiva, v. 20, n. 5, p. 1541-1547, 2015.

WORLD HEALTH ORGANIZATION - WHO. Control of leishmaniases. Geneva, 2010. (Technical Report Series, 949). 